\title{
Using a (Higher-Order) Magnus Method to Solve the Sturm-Liouville Problem
}

\author{
Veerle Ledoux ${ }^{\star}$, Marnix Van Daele, and Guido Vanden Berghe \\ Vakgroep Toegepaste Wiskunde en Informatica, Ghent University, \\ Krijgslaan 281-S9, B-9000 Gent, Belgium \\ \{Veerle.Ledoux, Marnix. VanDaele, Guido.VandenBerghe\}@UGent . be
}

\begin{abstract}
The main purpose of this paper is to describe techniques for the numerical solution of a Sturm-Liouville equation (in its Schrödinger form) by employing a Magnus expansion. With a suitable method to approximate the highly oscillatory integrals which appear in the Magnus series, high order schemes can be constructed. A method of order ten is presented. Even when the solution is highly-oscillatory, the scheme can accurately integrate the problem using stepsizes typically much larger than the solution "wavelength". This makes the method well suited to be applied in a shooting process to locate the eigenvalues of a boundary value problem.
\end{abstract}

\section{Introduction}

In this paper we are concerned with the numerical approximation of problems of the form

$$
y^{\prime \prime}(x)=[V(x)-E] y(x), \quad a \leq x \leq b
$$

This equation is the Sturm-Liouville equation in its Liouville normal form, also called Schrödinger form. Mathematically, Schrödinger problems arise from the standard separation of variables method applied to a linear partial differential equation, and in connection with the inverse scattering transform for solving nonlinear partial differential equations. The Schrödinger equation is also well known as the fundamental equation in quantum physics or quantum chemistry but arises for instance also in geophysical applications, and vibration and heat flow problems in mechanical engineering. Many Schrödinger problems have explicit solutions, and are therefore important in the analytic investigation of different physical models. However most (boundary value) problems cannot be solved analytically, and computationally efficient approximation techniques are of great applicability. Although we focus in this paper on the basic Schrödinger equation in a finite domain and with a smooth potential $V(x)$, our scheme can be extended to a more general Sturm-Liouville problem $-\left(p(x) y^{\prime}(x)\right)^{\prime}+q(x) y(x)=E w(x) y(x)$.

The parameter $E$ (also called the eigenvalue) in (11) is unknown, and is to be found subject to some kind of boundary conditions in the endpoints $a$ and $b$.

\footnotetext{
* Postdoctoral Fellow of the Fund for Scientific Research - Flanders (Belgium)
}

(F.W.O.-Vlaanderen).

M. Bubak et al. (Eds.): ICCS 2008, Part I, LNCS 5101, pp. 1032 -1041, 2008.

(C) Springer-Verlag Berlin Heidelberg 2008 
It is well known that as $E$ grows, the solutions of (1) become increasingly oscillatory. In fact, as $E \rightarrow+\infty$ the solution "wave length" approaches $2 \pi / \sqrt{E}$. This highly oscillatory character of the solution is the reason why standard integrators encounter difficulties in efficiently estimating the higher eigenvalues: a naive integrator will be forced to make increasingly smaller steps severely increasing the running time.

By taking advantage of special methods, one can construct numerical algorithms having special advantages over these standard (naive) methods. Pruess suggested to approximate the coefficients of the problem by piecewise constant approximations, solving the problem analytically on the piecewise constant intervals (see 1516]). For such a coefficient approximation method the step size is not restricted by the oscillations in the solution but the scheme is only second order, unless Richardson extrapolation approximations are made. Two approaches have been suggested to construct higher order schemes, both being natural extensions of the Pruess ideas. A first approach is based on a technique from mathematical physics: the perturbation approximation, leading to the so-called Piecewise Perturbation Methods (PPM) (see 891011]). In [2] it was shown that the piecewise perturbation approach may be viewed as the application of a modified Neumann series. The second approach consists in the application of another integral series: the Magnus series.

During the last decade, numerical schemes based on the Magnus expansion received a lot of attention due to their preservation of Lie group symmetries (see [5], 14], and references cited therein). More generally, Magnus methods have been applied in spectral theory, Hamiltonian systems, symplectic and unitary integration, control theory, stochastic systems, and quantum chemistry; see [1] for a list of applications. Moan [13] was the first to consider a Magnus method in the context of Sturm-Liouville problems. He applied a Magnus series integrator directly to eq. (1) with a piecewise polynomial $V(x)$. However poor approximations can then be expected for large eigenvalues. Later Degani and Schiff [23] and Iserles [4] showed that it is a better idea for oscillatory ordinary differential equations to apply the Magnus series integrator not directly to the equation but to the so-called modified equation. In 12 such a modified Magnus scheme of order eight was constructed for the Schrödinger problem and applied in a shooting procedure to compute the eigenvalues of the boundary value problem. In the current paper we present the construction of a modified Magnus method of order ten. In order to reach tenth order, the Filon-based quadrature rule for the oscillatory integrals appearing in the Magnus series, had to be extended to triple integrals. Also this new modified Magnus integrator can be used in a shooting process to efficiently compute eigenvalues.

\section{The (Modified) Magnus Method}

The differential equation (11) is converted into a system of first-order ODE's

$$
\mathbf{y}(x)^{\prime}=A(x, E) \mathbf{y}(x), \quad \mathbf{y}(a)=\mathbf{y}_{0},
$$


where

$$
A(x, E)=\left(\begin{array}{cr}
0 & 1 \\
V(x)-E & 0
\end{array}\right),
$$

and $\mathbf{y}=\left[y(x), y^{\prime}(x)\right]^{T}$. Suppose that we have already computed $\mathbf{y}_{i} \approx \mathbf{y}\left(x_{i}\right)$ and that we wish to advance the numerical solution to $x_{i+1}=x_{i}+h_{i}$. We first compute a constant approximation $\bar{V}$ of the potential function $V(x)$

$$
\bar{V}=\frac{1}{h_{i}} \int_{x_{i}}^{x_{i}+h_{i}} V(x) d x .
$$

Next we change the frame of reference by letting

$$
\mathbf{y}(x)=e^{\left(x-x_{i}\right) \bar{A}} \mathbf{u}\left(x-x_{i}\right), \quad x_{i} \leq x \leq x_{i+1}
$$

where

$$
\bar{A}(E)=\left(\begin{array}{cr}
0 & 1 \\
\bar{V}-E & 0
\end{array}\right) .
$$

We treat $\mathbf{u}$ as our new unknown which itself obeys the linear differential equation

$$
\mathbf{u}^{\prime}(\delta)=B(\delta, E) \mathbf{u}(\delta), \quad \delta=x-x_{i} \in\left[0, h_{i}\right], \quad \mathbf{u}(0)=\mathbf{y}_{i}
$$

where

$$
B(\delta, E)=e^{-\delta \bar{A}}\left(A\left(x_{i}+\delta\right)-\bar{A}\right) e^{\delta \bar{A}} .
$$

The matrix $B$ can be computed explicitly. With $\xi(Z)$ and $\eta_{0}(Z)$ defined as

$$
\begin{gathered}
\xi(Z)= \begin{cases}\cos \left(|Z|^{1 / 2}\right) & \text { if } Z \leq 0, \\
\cosh \left(Z^{1 / 2}\right) & \text { if } Z>0,\end{cases} \\
\eta_{0}(Z)= \begin{cases}\sin \left(|Z|^{1 / 2}\right) /|Z|^{1 / 2} & \text { if } Z<0, \\
1 & \text { if } Z=0, \\
\sinh \left(Z^{1 / 2}\right) / Z^{1 / 2} & \text { if } Z>0,\end{cases}
\end{gathered}
$$

we can write $B$ as

$$
B(\delta, E)=\Delta V(\delta)\left(\begin{array}{cc}
\delta \eta_{0}\left(Z_{2 \delta}\right) & \frac{1-\xi\left(Z_{2 \delta}\right)}{2(E-\bar{V})} \\
-\frac{1+\xi\left(Z_{2 \delta}\right)}{2} & -\delta \eta_{0}\left(Z_{2 \delta}\right)
\end{array}\right),
$$

where $\Delta V(\delta)=\bar{V}-V\left(x_{i}+\delta\right)$ and $Z_{\gamma}=Z(\gamma)=(\bar{V}-E) \gamma^{2}$. Note that the PPM-formulation in e.g. [8] uses the same functions $\xi(Z)$ and $\eta_{0}(Z)$.

We apply a Magnus method to the modified equation (7). The Magnus expansion is then (where the bracket denotes the matrix commutator)

$$
\sigma(\delta)=\sigma_{1}(\delta)+\sigma_{2}(\delta)+\sigma_{3}(\delta)+\sigma_{4}(\delta)+\ldots,
$$


where

$$
\begin{aligned}
& \sigma_{1}(\delta)=\int_{0}^{\delta} B(x) d x \\
& \sigma_{2}(\delta)=-\frac{1}{2} \int_{0}^{\delta} \int_{0}^{x_{1}}\left[B\left(x_{2}\right), B\left(x_{1}\right)\right] d x_{2} d x_{1} \\
& \sigma_{3}(\delta)=\frac{1}{12} \int_{0}^{\delta}\left[\int_{0}^{x_{1}} B\left(x_{2}\right) d x_{2},\left[\int_{0}^{x_{1}} B\left(x_{2}\right) d x_{2}, B\left(x_{1}\right)\right]\right] d x_{1}, \\
& \sigma_{4}(\delta)=\frac{1}{4} \int_{0}^{\delta}\left[\int_{0}^{x_{1}}\left[\int_{0}^{x_{2}} B\left(x_{3}\right) d x_{3}, B\left(x_{2}\right)\right] d x_{2}, B\left(x_{1}\right)\right] d x_{1},
\end{aligned}
$$

and $\mathbf{u}(\delta)=e^{\sigma(\delta)} \mathbf{y}_{i}, \delta \geq 0$. Thus, to compute $\mathbf{y}_{i+1}=e^{h \bar{A}} e^{\sigma(h)} \mathbf{y}_{i}$ with $h=h_{i}$, we need to approximate $\sigma(h)$ by truncating the expansion (12) and replacing integrals by quadrature (see next section). The $2 \times 2$ matrix exponentials $e^{h \bar{A}}$ and $e^{\sigma(h)}$ can be written down explicitely. $e^{h \bar{A}}$ is the matrix exponential of a constant matrix, and thus

$$
\operatorname{expm}\left(\begin{array}{cc}
0 & h \\
h(\bar{V}-E) & 0
\end{array}\right)=\left(\begin{array}{cc}
\xi\left(Z_{h}\right) & h \eta_{0}\left(Z_{h}\right) \\
Z_{h} \eta_{0}\left(Z_{h}\right) / h & \xi\left(Z_{h}\right)
\end{array}\right), \quad Z_{h}=Z(h) .
$$

To write down an expression for $e^{\sigma(h)}$, we note that $\sigma(h)$ is always a two by two matrix with zero trace. For such matrices the following is true:

$$
\operatorname{expm}\left(\begin{array}{cc}
a & b \\
c-a
\end{array}\right)=\left(\begin{array}{cc}
\xi(\omega)+a \eta_{0}(\omega) & b \eta_{0}(\omega) \\
c \eta_{0}(\omega) & \xi(\omega)-a \eta_{0}(\omega)
\end{array}\right), \quad \omega=a^{2}+b c
$$

Here $a, b, c, \omega$ are functions of $x$ and $E$.

\section{Integration of the Integrals}

As shown in [4, the regular Magnus quadrature formulae (7]) are useless in the presence of high oscillation. For $E \gg \bar{V}$ the matrix function $B$ in (11) is highly oscillatory and quadrature must be used that respects high oscillation. Filon-type quadrature can be used to approximate highly oscillating integrals to a suitable precision in a small number of function evaluations per step. As in [12, we will apply Filon-type quadrature not-only in the oscillatory region $E>\bar{V}$, but also in the nonoscillatory $E<V(x)$ region (where it is just as good as regular Gauss-Christoffel Magnus quadrature).

The univariate Filon-rule is discussed in 4 and has the nice property that while regular quadrature is ineffective in the presence of high oscillation, Filon quadrature delivers accuracy which actually improves with higher oscillation. Here, we use this Filon-rule to approximate the univariate (modified) Magnus integral $\int_{0}^{h} B(\delta) d \delta$. In fact, this means that $\Delta V(\delta)$ in (11) is replaced by the Lagrange polynomial $\mathfrak{L}_{\Delta V}(\delta)=\sum_{k=1}^{\nu} \Delta V\left(c_{k} h\right) \ell_{k}(\delta)$ where $\ell_{k}$ is the $k$ th cardinal polynomial of Lagrangian interpolation and $c_{1}, c_{2}, \ldots, c_{\nu}$ are distinct quadrature 
nodes. The resulting integrals can then be solved analytically. An alternative way to obtain the interpolating polynomial $\mathfrak{L}_{\Delta V}(\delta)$ is by approximating $V(x)$ by a series over shifted Legendre polynomials:

$$
V(x) \approx \sum_{s=0}^{\nu-1} V_{s} h^{s} P_{s}^{*}(\delta / h)
$$

By the method of least squares the expressions for the coefficients $V_{s}$ are obtained:

$$
V_{s}=\frac{(2 s+1)}{h^{s+1}} \int_{0}^{h} V\left(x_{i}+\delta\right) P_{s}^{*}(\delta / h) d \delta, \quad m=0,1,2, \ldots
$$

It can then be noted that $\bar{V}=V_{0}$ and $\Delta V(\delta) \approx \mathfrak{L}_{\Delta V}(\delta)=-\sum_{s=1}^{\nu-1} V_{s} h^{s} P_{s}^{*}(\delta / h)$. To compute the integrals (16) tenth-order Gauss-Legendre is used, requiring $\nu=5$ function evaluations of $V$ (Gauss-Lobatto is another option).

With

$$
\widehat{\xi}=\xi\left(Z_{2 h}\right), \quad \widehat{\eta}_{0}=\eta_{0}\left(Z_{2 h}\right), \quad Z_{2 h}=4 Z_{h}=4(\bar{V}-E) h^{2}
$$

and $\hat{V}_{s}=h^{s+1} V_{s}, s=1, \ldots, 4$, we obtain then the following

$$
\begin{aligned}
\frac{1}{h} \int_{0}^{h} \Delta V(\delta) \delta \eta_{0}\left(Z_{2 \delta}\right) d \delta \approx & \frac{\left(\hat{V}_{1} / 2+5 \hat{V}_{4}+3 \hat{V}_{2} / 2+3 \hat{V}_{3}\right) \widehat{\eta}_{0}}{Z_{h}} \\
& +\frac{\left(-\hat{V}_{3}-\hat{V}_{2}-\hat{V}_{4}-\hat{V}_{1}\right) \hat{\xi}-\hat{V}_{1}+\hat{V}_{4}+\hat{V}_{2}-\hat{V}_{3}}{4 Z_{h}} \\
& +\frac{\left(-45 \hat{V}_{4}-3 \hat{V}_{2}-15 \hat{V}_{3}\right) \hat{\xi}-15 \hat{V}_{3}+45 \hat{V}_{4}+3 \hat{V}_{2}}{4 Z_{h}^{2}} \\
& +\frac{\left(15 \hat{V}_{3}+105 \hat{V}_{4}\right) \widehat{\eta}_{0}}{2 Z_{h}^{2}}+\frac{-105 \hat{V}_{4} / 4 \hat{\xi}+105 \hat{V}_{4} / 4}{Z_{h}^{3}} \\
\int_{0}^{h} \Delta V(\delta)\left(1+\xi\left(Z_{2 \delta}\right)\right) d \delta \approx & \int_{0}^{h} \Delta V(\delta) \xi\left(Z_{2 \delta}\right) d \delta \\
\int_{0}^{h} \Delta V(\delta)\left(1-\xi\left(Z_{2 \delta}\right)\right) d \delta \approx & -\int_{0}^{h} \Delta V(\delta) \xi\left(Z_{2 \delta}\right) d \delta \\
\approx & \left(\hat{V}_{1}+\hat{V}_{2}+\hat{V}_{3}+\hat{V}_{4}\right) \widehat{\eta}_{0}+\frac{\left(3 \hat{V}_{2}+15 \hat{V}_{3}+45 \hat{V}_{4}\right) \widehat{\eta}_{0}}{Z_{h}} \\
& +\frac{\left(-3 \hat{V}_{2}-\hat{V}_{1}-10 \hat{V}_{4}-6 \hat{V}_{3}\right) \hat{\xi}+6 \hat{V}_{3}-3 \hat{V}_{2}-10 \hat{V}_{4}+\hat{V}_{1}}{2 Z_{h}} \\
& +\frac{210 \hat{V}_{4} \widehat{\eta}_{0}+\left(-105 \hat{V}_{4}-15 \hat{V}_{3}\right) \hat{\xi}-105 \hat{V}_{4}+15 \hat{V}_{3}}{2 Z_{h}^{2}} \quad(18)
\end{aligned}
$$

which allows us to approximate $\int_{0}^{h} B(\delta) d \delta$. Including only this first Magnusterm is sufficient to have a fourth-order method. However to construct a method 
of order ten, we need to include more Magnus terms. First we consider the approximation of $\sigma_{2}$. We extend the Filon idea to the computation of the double integral. As in $[12$ we write the double integral as

$$
\begin{aligned}
\int_{0}^{h} \int_{0}^{\delta_{1}}\left[B\left(\delta_{2}\right), B\left(\delta_{1}\right)\right] d \delta_{2} d \delta_{1} & =2 \int_{0}^{h} \int_{0}^{\delta_{1}} \Delta V\left(\delta_{1}\right) \Delta V\left(\delta_{2}\right) K_{1}\left(\delta_{1}, \delta_{2}\right) d \delta_{2} d \delta_{1} U_{1} \\
& +2 \int_{0}^{h} \int_{0}^{\delta_{1}} \Delta V\left(\delta_{1}\right) \Delta V\left(\delta_{2}\right) K_{2}\left(\delta_{1}, \delta_{2}\right) d \delta_{2} d \delta_{1} U_{2} \\
& +2 \int_{0}^{h} \int_{0}^{\delta_{1}} \Delta V\left(\delta_{1}\right) \Delta V\left(\delta_{2}\right) K_{3}\left(\delta_{1}, \delta_{2}\right) d \delta_{2} d \delta_{1} U_{3}
\end{aligned}
$$

where $K_{1}(x, y)=y \eta_{0}\left(Z_{2 y}\right)-x \eta_{0}\left(Z_{2 x}\right), K_{2}(x, y)=\xi\left(Z_{2 x}\right)-\xi\left(Z_{2 y}\right), K_{3}(x, y)=$ $(x-y) \eta_{0}\left(Z_{2(x-y)}\right)$ and

$$
U_{1}=\left(\begin{array}{cc}
0 & \frac{1}{2(E-V)} \\
\frac{1}{2} & 0
\end{array}\right), \quad U_{2}=\left(\begin{array}{cc}
-\frac{1}{4(E-V)} & 0 \\
0 & \frac{1}{4(E-V)}
\end{array}\right), \quad U_{3}=\left(\begin{array}{cc}
0 & \frac{1}{2(E-V)} \\
\frac{-1}{2} & 0
\end{array}\right) .
$$

The three integrals in (19) must be replaced by quadrature. We again replace $\Delta V$ by the polynomial $\mathfrak{L}_{\Delta V}$ and solve the resulting integrals analytically (Maple). For brevity reasons we do not list the full expressions of the resulting formulae here, we show only the expression for the third integral:

$$
\begin{aligned}
\int_{0}^{h} & \int_{0}^{\delta_{1}} \Delta V\left(\delta_{1}\right) \Delta V\left(\delta_{2}\right) K_{3}\left(\delta_{1}, \delta_{2}\right) d \delta_{2} d \delta_{1} \approx \\
& \left(\frac{\hat{V}_{2}^{2}+\hat{V}_{4}^{2}-\hat{V}_{3}^{2}-\hat{V}_{1}^{2}+2\left(\hat{V}_{4} \hat{V}_{2}-\hat{V}_{3} \hat{V}_{1}\right)}{4 Z_{h}}+\frac{190 \hat{V}_{4}^{2}-\hat{V}_{1}^{2}+15 \hat{V}_{2}^{2}-66 \hat{V}_{3}^{2}}{4 Z_{h}^{2}}\right. \\
& +\frac{-42 \hat{V}_{3} \hat{V}_{1}+156 \hat{V}_{4} \hat{V}_{2}}{4 Z_{h}^{2}}+\frac{9 \hat{V}_{2}^{2}-405 \hat{V}_{3}^{2}+4335 \hat{V}_{4}^{2}-30 \hat{V}_{3} \hat{V}_{1}+1110 \hat{V}_{4} \hat{V}_{2}}{4 Z_{h}^{3}} \\
& \left.+\frac{-225 \hat{V}_{3}^{2}+20475 \hat{V}_{4}^{2}+630 \hat{V}_{4} \hat{V}_{2}}{4 Z_{h}^{4}}+\frac{11025 \hat{V}_{4}^{2}}{4 Z_{h}^{5}}\right) \hat{\eta}_{0}+\left(\frac{\hat{V}_{1}^{2}-3 \hat{V}_{2}^{2}+6 \hat{V}_{3}^{2}-10 \hat{V}_{4}^{2}}{4 Z_{h}^{2}}\right. \\
& +\frac{7 \hat{V}_{3} \hat{V}_{1}-13 \hat{V}_{4} \hat{V}_{2}}{4 Z_{h}^{2}}+\frac{-1110 \hat{V}_{4}^{2}-270 \hat{V}_{4} \hat{V}_{2}+30 \hat{V}_{3} \hat{V}_{1}-9 \hat{V}_{2}^{2}+105 \hat{V}_{3}^{2}}{4 Z_{h}^{3}} \\
& \left.+\frac{225 \hat{V}_{3}^{2}-630 \hat{V}_{4} \hat{V}_{2}-5775 \hat{V}_{4}^{2}}{4 Z_{h}^{4}}-\frac{11025 \hat{V}_{4}^{2}}{4 Z_{h}^{5}}\right) \hat{\xi} \\
& +\frac{-\hat{V}_{2}^{2} / 20-\hat{V}_{1}^{2} / 12-\hat{V}_{3}^{2} / 28-\hat{V}_{4}^{2} / 36}{Z_{h}}+\frac{-7 \hat{V}_{4} \hat{V}_{2}-5 \hat{V}_{3} \hat{V}_{1}}{4 Z_{h}^{2}} .
\end{aligned}
$$

As shown in 12 the inclusion of this second Magnus term leads to an eighthorder algorithm. Next we consider the approximation of $\sigma_{3}$ and $\sigma_{4}$ in order to have a tenth-order scheme. The same procedure is applied again: the function 
$\Delta V$ appearing in the expressions for $\sigma_{3}$ and $\sigma_{4}$ is replaced by a polynomial. By symbolic computation it can be shown that it is sufficient here to replace $\Delta V(\delta)$ by a third-degree polynomial. Therefore we take $\Delta V(\delta) \approx \sum_{s=1}^{3} V_{s} h^{s} P_{s}^{*}(\delta / h)$, where the coefficients $V_{s}$ are still the same as the ones before. Also only the terms where the degree in $h$ is smaller than 11 have to be considered: e.g. we do not take into account the $\hat{V}_{3}^{3}$-term. We used the symbolic software package Maple to compute the expressions of the 2 by 2 matrix $\varsigma=\sigma_{3}+\sigma_{4}$. As an illustration, we show some terms of the diagonal elements:

$$
\begin{aligned}
\varsigma_{11}=-\varsigma_{22} & =\left(\frac{135 \hat{V}_{1}^{2} \hat{V}_{2}+49 \hat{V}_{2}^{3}+240 \hat{V}_{1} \hat{V}_{3} \hat{V}_{2}+45 \hat{V}_{1}^{3}+150 \hat{V}_{1}^{2} \hat{V}_{3}+123 \hat{V}_{1} \hat{V}_{2}^{2}}{480 Z_{h}^{2}}\right. \\
& +\frac{961 \hat{V}_{1}^{2} \hat{V}_{2}+105 \hat{V}_{1}^{3}+8382 \hat{V}_{1} \hat{V}_{3} \hat{V}_{2}+2475 \hat{V}_{1}^{2} \hat{V}_{3}+2025 \hat{V}_{1} \hat{V}_{2}^{2}+1161 \hat{V}_{2}^{3}}{96 Z_{h}^{3}} \\
& +\frac{5859 \hat{V}_{1} \hat{V}_{2}^{2}+59662 \hat{V}_{1} \hat{V}_{3} \hat{V}_{2}+7245 \hat{V}_{1}^{2} \hat{V}_{3}+8055 \hat{V}_{2}^{3}+736 \hat{V}_{1}^{2} \hat{V}_{2}}{32 Z_{h}^{4}} \\
& \left.+\frac{549 \hat{V}_{2}^{3}+16305 \hat{V}_{1} \hat{V}_{3} \hat{V}_{2} / 4}{Z_{h}^{5}}\right) \hat{\xi}+\ldots
\end{aligned}
$$

The formulas in (17), (21) and (22) may be problematic for $E$ close to $\bar{V}$ due to near-cancellation of like terms. Therefore alternative formulas are used for small $Z_{h}$ values (see [12]). These alternative formulas are obtained by applying a Taylor expansion. The alternative for expression (17) is then e.g.

$$
\begin{aligned}
& \frac{1}{h} \int_{0}^{h} \Delta V(\delta) \delta \eta_{0}\left(Z_{2 \delta}\right) d \delta \approx \\
& \left(\hat{V}_{1} / 3+\hat{V}_{2} / 15\right) Z_{h}+\left(\hat{V}_{3} / 105+4 \hat{V}_{2} / 105+4 \hat{V}_{1} / 45+\hat{V}_{4} / 945\right) Z_{h}^{2} \\
& +\left(2 \hat{V}_{3} / 945+\hat{V}_{2} / 189+\hat{V}_{1} / 105+2 \hat{V}_{4} / 3465\right) Z_{h}^{3}+\ldots
\end{aligned}
$$

The alternative formulae are used in the interval $\left|Z_{h}\right|<0.15$, in this case it is found that it is sufficient to go up to $Z_{h}^{8}$.

\section{Shooting for Eigenvalues}

As mentioned before, a shooting procedure can be used to locate the eigenvalues of the boundary value problem associated with (1). The modified Magnus method presented here is well suited for the repeated solution of the initial value problems which appear in the shooting procedure. These initial value problems are solved for a fixed potential $V$ but for different values of $E$. For our modified Magnus integrator, a mesh can be constructed which only depends on $V$ and not on $E$ (a procedure similar as in 12 can be used to construct the mesh). This mesh has to be computed only once and is then used in all eigenvalue computations. Moreover the value $\bar{V}$ and the coefficients $V_{s}$ are computed and 


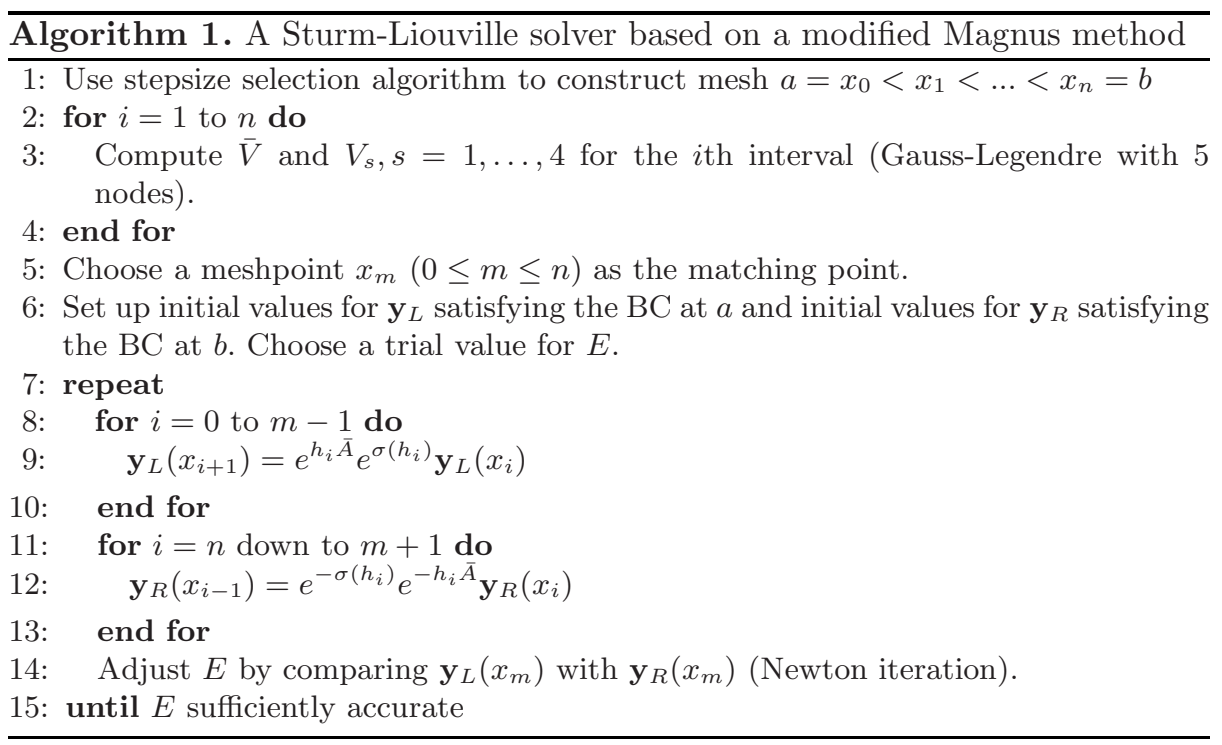

stored once for all before the start of the shooting process. Algorithm 1 shows the basic shooting procedure in which the modified Magnus algorithm is used to propagate the left-hand and right-hand solutions. For more details on such a shooting procedure we refer to [12].

\section{$5 \quad$ Numerical Examples}

As test potentials we take two well-known test problems from the literature [17. The Coffey-Evans problem is a Schrödinger equation with

$$
V(x)=-2 \beta \cos (2 x)+\beta^{2} \sin ^{2}(2 x)
$$

and $y(-\pi / 2)=y(\pi / 2)=0$ as boundary conditions. Here we take $\beta=30$. The second problem is the Woods-Saxon problem defined by

$$
V(x)=-50 \frac{1-\frac{5 t}{3(1+t)}}{1+t}
$$

with $t=e^{(x-7) / 0.6}$ over the interval $[0,15]$. The eigenvalue spectrum of this Woods-Saxon problem contains 14 eigenenergies $E_{0}, \ldots, E_{13}$. We take here an equidistant mesh. Note however that an automatic stepsize selection algorithm can be constructed as in [12. We performed some eigenvalue computations at different step lengths. The absolute errors $\Delta E_{k}=E_{k}^{\text {exact }}-E_{k}^{\text {comput }}$ are collected in Table 1. For the Coffey-Evans problem some lower eigenvalues come in very close clusters and to distinguish between them the search algorithm must rely on a highly accurate integrator. Our modified Magnus method deals very well with these close eigenvalues. Also no systematic deterioration of the accuracy is 
Table 1. Absolute value of (absolute) errors $\Delta E_{k}$ for the Coffey-Evans and WoodsSaxon problem. $n$ is the number of (equidistant) steps. $a \mathrm{E}-b$ means $a \cdot 10^{-b}$.

\begin{tabular}{crccc|cccc}
\hline \multicolumn{4}{c}{ Coffey-Evans problem } \\
$k$ & $E_{k}$ & $n=128$ & $n=256$ & $k$ & $E_{k}$ & $n=64$ & $n=128$ \\
\hline 0 & 0.0000000000000000 & $3.4 \mathrm{E}-10$ & $2.2 \mathrm{E}-13$ & 0 & -49.45778872808258 & $3.9 \mathrm{E}-11$ & $8.5 \mathrm{E}-14$ \\
1 & 117.9463076620687587 & $1.5 \mathrm{E}-9$ & $1.4 \mathrm{E}-12$ & 1 & -48.14843042000639 & $3.8 \mathrm{E}-10$ & $2.6 \mathrm{E}-13$ \\
2 & 231.6649292371271088 & $2.1 \mathrm{E}-9$ & $1.1 \mathrm{E}-12$ & 2 & -46.29075395446623 & $2.0 \mathrm{E}-9$ & $1.6 \mathrm{E}-12$ \\
3 & 231.6649293129610125 & $1.1 \mathrm{E}-9$ & $1.1 \mathrm{E}-12$ & 3 & -43.96831843181467 & $7.2 \mathrm{E}-9$ & $6.3 \mathrm{E}-12$ \\
4 & 231.6649293887949167 & $2.1 \mathrm{E}-9$ & $7.9 \mathrm{E}-13$ & 4 & -41.23260777218090 & $2.0 \mathrm{E}-8$ & $1.9 \mathrm{E}-12$ \\
5 & 340.8882998096130157 & $4.5 \mathrm{E}-9$ & $4.4 \mathrm{E}-12$ & 5 & -38.12278509672854 & $4.8 \mathrm{E}-8$ & $4.6 \mathrm{E}-11$ \\
6 & 445.2830895824354620 & $4.4 \mathrm{E}-9$ & $3.6 \mathrm{E}-12$ & 6 & -34.67231320569997 & $9.7 \mathrm{E}-8$ & $9.7 \mathrm{E}-11$ \\
8 & 445.2832550313310036 & $4.4 \mathrm{E}-9$ & $2.7 \mathrm{E}-12$ & 7 & -30.91224748790910 & $1.7 \mathrm{E}-7$ & $1.7 \mathrm{E}-10$ \\
10 & 637.6822498740469991 & $4.8 \mathrm{E}-9$ & $4.2 \mathrm{E}-12$ & 8 & -26.87344891605993 & $2.8 \mathrm{E}-7$ & $2.9 \mathrm{E}-10$ \\
15 & 802.4787986926240517 & $2.8 \mathrm{E}-9$ & $1.7 \mathrm{E}-12$ & 9 & -22.58860225769320 & $3.9 \mathrm{E}-7$ & $4.3 \mathrm{E}-10$ \\
20 & 951.8788067965913828 & $2.3 \mathrm{E}-9$ & $3.7 \mathrm{E}-12$ & 10 & -18.09468828212811 & $5.1 \mathrm{E}-7$ & $5.7 \mathrm{E}-10$ \\
30 & 1438.2952446408023577 & $2.0 \mathrm{E}-9$ & $2.5 \mathrm{E}-12$ & 11 & -13.43686904026007 & $5.9 \mathrm{E}-7$ & $6.7 \mathrm{E}-10$ \\
40 & 2146.4053605398535082 & $1.5 \mathrm{E}-9$ & $2.7 \mathrm{E}-12$ & 12 & -8.67608167074520 & $6.0 \mathrm{E}-7$ & $7.2 \mathrm{E}-10$ \\
50 & 3060.9234915114205911 & $1.0 \mathrm{E}-9$ & $2.7 \mathrm{E}-12$ & 13 & -3.90823248120989 & $5.0 \mathrm{E}-7$ & $6.6 \mathrm{E}-10$ \\
\hline
\end{tabular}

observed as $k$ is increased. This tenth-order method gives of course more accurate approximations than the eighth order method of [12]: this method gives e.g. for the first eigenvalue of the Coffey-Evans problem an error of 1.0E-7 $(n=128)$ and $4.0 \mathrm{E}-10(n=256)$.

\section{Conclusion}

In this paper we discussed a modified Magnus method of order ten for the integration of a Sturm-Liouville problem in the Schrödinger form. Therefore the modified Magnus method described earlier by Degani and Schiff and Iserles had to be extended to the non-oscillatory $E<V$ region and a Filon-like quadrature rule had to be defined for the multivariate integrals appearing in the Magnus series. The modified Magnus method can be applied in a shooting procedure in order to compute the eigenvalues of a boundary value problem. Since an $E$-independent mesh can be constructed, all function evaluations can be done before the actual shooting process, which makes the method well suited to compute large batches of eigenvalues or just particularly large eigenvalues.

\section{References}

1. Blanes, S., Casas, F., Oteo, J.A., Ros, J.: Magnus and Fer expansions for matrix differential equations: the convergence problems. J. Phys A: Math. Gen. 31, 259268 (1998)

2. Degani, I., Schiff, J.: RCMS: Right Correction Magnus Series approach for oscillatory ODEs. J. Comput. Appl. Math. 193, 413-436 (2006) 
3. Degani, I.: RCMS - Right Correction Magnus Schemes for oscillatory ODEs and cubature formulae and commuting extensions. Thesis (PhD). Weizmann Institute of Science (2004)

4. Iserles, A.: On the numerical quadrature of highly oscillatory integrals I: Fourier transforms. IMA J. Numer. Anal. 24, 365-391 (2004)

5. Iserles, A., Nørsett, S.P.: On the solution of linear differential equations in Lie groups. Phil. Trans. R. Soc. Lond. A. 357, 983-1019 (1999)

6. Iserles, A.: On the global error of discretization methods for highly-oscillatory ordinary differential equations. BIT 42, 561-599 (2002)

7. Iserles, A., Munthe-Kaas, H.Z., Nørsett, S.P., Zanna, A.: Lie-group methods. Acta Numerica 9, 215-365 (2000)

8. Ixaru, L.G.: Numerical Methods for Differential Equations and Applications. Reidel, Dordrecht-Boston-Lancaster (1984)

9. Ixaru, L.G., De Meyer, H., Vanden Berghe, G.: SLCPM12 - A program for solving regular Sturm-Liouville problems. Comp. Phys. Commun. 118, 259-277 (1999)

10. Ledoux, V., Van Daele, M., Vanden Berghe, G.: CP methods of higher order for Sturm-Liouville and Schrödinger equations. Comput. Phys. Commun. 162, 151-165 (2004)

11. Ledoux, V., Van Daele, M., Vanden Berghe, G.: MATSLISE: A MATLAB package for the Numerical Solution of Sturm-Liouville and Schrödinger equations. ACM Trans. Math. Software 31, 532-554 (2005)

12. Ledoux, V., Van Daele, M., Vanden Berghe, G.: Efficient numerical solution of the 1D Schrödinger eigenvalue problem using Magnus integrators. IMA J. Numer. Anal (submitted)

13. Moan, P.C.: Efficient approximation of Sturm-Liouville problems using Lie group methods. Technical report. DAMTP University of Cambridge (1998)

14. Munthe-Kaas, H., Owren, B.: Computations in a free Lie algebra, Phil. Trans. R. Soc. Lond. A. 357, 957-981 (1999)

15. Pruess, S.: Solving linear boundary value problems by approximating the coefficients. Math. Comp. 27, 551-561 (1973)

16. Pruess, S., Fulton, C.T.: Mathematical software for Sturm-Liouville problems. ACM Trans. on Math. Software 19, 360-376 (1993)

17. Pryce, J.D.: Numerical Solution of Sturm-Liouville Problems. Clarendon Press (1993) 